\title{
Pulsed Laser Heating of a Finite Silver Selenide Slab Using (HHCE) Model
}

\author{
Mohamed Abdelhady Kamel EL-Adawi, Safaa Abdelfattah Shalaby \\ Physics Department, Faculty of Education, Ain Shams University, Heliopolis, Cairo, Egypt \\ Email: adawish1@hotmail.com
}

How to cite this paper: EL-Adawi, M.A.K. and Shalaby, S.A. (2018) Pulsed Laser Heating of a Finite Silver Selenide Slab Using (HHCE) Model. Applied Mathematics, 9, 355-368.

https://doi.org/10.4236/am.2018.94027

Received: February 22, 2018

Accepted: April 21, 2018

Published: April 24, 2018

Copyright () 2018 by authors and Scientific Research Publishing Inc. This work is licensed under the Creative Commons Attribution International License (CC BY 4.0).

http://creativecommons.org/licenses/by/4.0/

\begin{abstract}
Heat induced in a finite Silver Selenide slab by an external pulsed laser source is studied in dimensionless scale according to hyperbolic heat conduction model (HHCE) using Laplace integral transform technique. The temperature profile, the critical time required to initiate phase transition and that to initiate damage at the front surface are obtained for different pulses and are illustrated graphically.
\end{abstract}

\section{Keywords}

Laser Heating, Hyperbolic Heat Conduction Equation, Laser Induced Phase Transition, Heating Silver Selenide Slab

\section{Introduction}

Heating sources of high power densities such as laser sources and microwaves have arisen recently an increasing interest of many investigators [1]. Such heating interaction has vital applications in different fields related to material processing such as spot wilding, laser cutting, surface annealing and drilling of metals [1] [2] [3] [4] [5]. In semiconductor industry, many other applications are developed including both local diffusion and alloying to form $\mathrm{p}-\mathrm{n}$ junctions. The most spectacular effects involve a change of phase of the absorbing material [1] [6] [7] [8] [9]. In many applications, it is particularly important to reveal the temperature distribution within the irradiated target, and the time required to initiate phase transition and to initiate melting.

Many authors assess theoretically such temperature profiles solving the heat conduction equation [3] [6] [8]-[22].

In principal, there are two trends concerning this point. One trend assumes that heat propagates with infinite velocity through the heated target (Parabolic 
heat conduction equation (PHCE)). The other trend suggests that heat propagates with finite velocity leading to the hyperbolic heat conduction equation (HHCE) [23] [24] [25] [26] [27] especially when extremely short laser pulse or very high frequencies are concerned. The existence of thermal waves propagating with a finite speed has been demonstrated experimentally [28] [29]. Several analytic and numerical solutions of such problems have been reported [23] [24] [25] [26] [30] [31] [32].

The present article represents a study of the problem of laser heating of a homogeneous finite slab of Silver Selenide $\left(\mathrm{Ag}_{2} \mathrm{Se}\right)$ material considering the hyperbolic heat conduction equation (HHCE). $\mathrm{Ag}_{2} \mathrm{Se}$ compound is of great technological importance as a promising thermoelectric power generator material [33], it has also applications in the field of the switching devices [34] [35]. Also it is well known that silver selenide $\mathrm{Ag}_{2} \mathrm{Se}$ undergoes a polymorphic phase transition [34]. It has two phases one below 400 degree Kelvin and is identified as $\beta-\mathrm{Ag}_{2} \mathrm{Se}$ phase with orthorhombic structure, the other phase is at higher temperatures and is called $\alpha-\mathrm{Ag}_{2} \mathrm{Se}$ phase with body-centered-cubic (bcc) form.

Moreover, silver selenide material shows semiconducting nature up to 403 degree Kelvin and then it shows a metallic nature with rise in temperature [34].

It is observed [34] that it undergoes this phase transition at $(403 \pm 2)$ degree Kelvin.

This alpha-beta transition temperature does depend on the pressure [36]. Moreover, it is revealed [37] that the phase transition temperature is affected by the heating rate and that it increases with a decrease of its thickness.

The aim of the present trial is to find analytically the temperature field within an $\mathrm{Ag}_{2} \mathrm{Se}$-slab subjected to a pulsed laser as a heating source. This makes it possible to determine the time required to initiate phase transition in such material, and the critical time required to initiate melting at the irradiated front surface of the considered slab. The problem is formulated in the light of the hyperbolic heat conduction equation $(\mathrm{HHCH})$ in dimensionless form. Laplace integral transform technique is considered to get the required solutions. Computations on silver selenide slab are given as an illustrative example.

\section{Mathematical Formulation}

In sitting up the problem a finite homogeneous slab of silver selenide $\mathrm{Ag}_{2} \mathrm{Se}$ material is heated using laser flux of power density $q(t), \mathrm{W} \cdot \mathrm{m}^{2}$ incident perpendicularly on its front surface. It is assumed that a part of the incident laser flux will be reflected at the front surface and a part will be absorbed by the target material.

It is also assumed that heat losses due to convection and thermal radiations are neglected [37]. The physical properties of the slab material are assumed to be temperature independent [37]. Several papers have been written on the thermoelectric properties, the thermal conductivity, Seebeck coefficient and the resistivity of silver selenide [33] [38]. The values $\lambda(T)$ obtained in [33] revealed that the thermal conductivity $\lambda(T)$ increases monotonously with increasing temperature such that $\Delta \lambda / \Delta T \approx 7.76 \times 10^{-7} \mathrm{~W} \cdot \mathrm{m} \cdot \mathrm{K}^{2}$ [33] [39]. 
This weak temperature dependence makes it possible to accept the assumption that its thermal conductivity is temperature independent for the considered temperature intervals [39]. The heat flow for our case is considered to be one dimensional [1] [2]. The $\mathrm{x}$-axis normal to the free surface of the slab is taken in the direction of the incident laser beam. The hyperbolic heat conduction equation (HHCE) is considered according to which the thermal effects propagate within the target with a finite velocity and thus a thermal relaxation time is involved in the related equations.

For such a case the modified Fourier equation (Cattaneo equation) is considered in the form:

$$
t_{k} \frac{\partial q(x, t)}{\partial t}+q(x, t)=-\lambda \nabla T(x, t)
$$

where $t_{k}=\frac{\alpha}{W^{2}}$ is the thermal relaxation time

$\alpha=\frac{\lambda}{\rho c_{p}}$ is the thermal diffusivity in terms of the thermal conductivity $\lambda$, $\mathrm{W} \cdot \mathrm{m} \cdot \mathrm{K}$, the density $\rho, \mathrm{kg} / \mathrm{m}^{3}$, the specific heat $c_{p}, \mathrm{j} / \mathrm{kg} \cdot \mathrm{K}$, and $W, \mathrm{~m} / \mathrm{sec}$ is the speed of the propagation of the thermal wave in the medium.

Equation (1) with the following conservation energy equation:

$$
-\nabla \cdot q=\rho c_{p} \frac{\partial T}{\partial t}
$$

Leads to the hyperbolic heat conduction equation (HHCE) in the form:

$$
t_{k} \frac{\partial^{2} T}{\partial t^{2}}+\frac{\partial T}{\partial t}=\alpha \nabla^{2} T
$$

Considering the following dimensionless scales:

$$
\begin{aligned}
X & =\frac{W}{2 \alpha} x \\
Y & =\frac{W}{2 \alpha} y \\
Z & =\frac{W}{2 \alpha} Z \\
\theta & =\frac{T-T_{0}}{T_{m}-T_{0}} \\
\varphi & =\frac{q(0, t)(1-R)}{W \rho c_{p}\left(T_{m}-T_{0}\right)}
\end{aligned}
$$

where,

$T_{0}$ is the ambient temperature, $T_{m}$ the melting temperature, and $R$ is the reflectivity at the front surface.

One obtains Cattaneo equation in dimensionless form as:

$$
\frac{\partial \varphi}{\partial \tau}+2 \varphi=-\nabla \theta, \quad \tau=\frac{t}{t_{k}}
$$


the energy conservation equation in the dimensionless scale attains the form:

$$
\frac{\partial \theta}{\partial \tau}=-\nabla \varphi
$$

and the hyperbolic heat conduction equation (HHCE) in the following dimensionless form is:

$$
\frac{\partial^{2} \theta}{\partial \tau^{2}}+2 \frac{\partial \theta}{\partial \tau}=\frac{\partial^{2} \theta}{\partial X^{2}}
$$

Equation (12) is subjected to the following dimensionless initial and boundary conditions:

$$
\begin{gathered}
\text { at } \tau=0, \quad \theta(X, 0)=0 \\
\text { at } X=X_{d}, \quad \frac{\partial \theta}{\partial X}\left(X_{d}, \tau\right)=0 \\
\text { at } X=0, \quad \frac{\partial \theta}{\partial X}(0, \tau)=-\left[2 \varphi(0, \tau)+\frac{\partial \varphi}{\partial \tau}(0, \tau)\right]
\end{gathered}
$$

Equation (14) indicates that the rear surface of the target is insulated.

Laplace integral transform technique is applied to solve Equation (12). Taking Laplace transform on Equation (12) w.r.t. the time $\tau$ one gets:

$$
\frac{\partial^{2}}{\partial X^{2}} \bar{\theta}(X, s)-\left(s^{2}+2 s\right) \bar{\theta}(X, s)=0
$$

The solution of equation (16) is as follows:

$$
\bar{\theta}(X, s)=A \mathrm{e}^{\sqrt{s(s+2)} X}+B \mathrm{e}^{-\sqrt{s(s+2)} X}
$$

at $X=0$ one gets:

$$
\left.\frac{\partial \bar{\theta}}{\partial X}\right|_{X=0}=A \sqrt{s(s+2)}-B \sqrt{s(s+2)}
$$

And at $X=X_{d}$ one gets:

$$
\begin{aligned}
& \left.\frac{\partial \bar{\theta}}{\partial X}\right|_{X=X_{d}}=A \sqrt{s(s+2)} \mathrm{e}^{\sqrt{s(s+2)} X_{d}}-B \sqrt{s(s+2)} \mathrm{e}^{-\sqrt{s(s+2)} X_{d}}=0 \\
& \text { Thus: } A \mathrm{e}^{\sqrt{s(s+2)} X_{d}}-B \mathrm{e}^{-\sqrt{s(s+2)} X_{d}}=0
\end{aligned}
$$

Taking Laplace transform to the boundary condition Equation (15) one gets:

$$
\frac{\partial \bar{\theta}}{\partial X}(X, s)=-[2 \bar{\varphi}(X, s)+\{s \bar{\varphi}(X, s) \varphi(X, 0)\}],
$$

At the front surface $X=0$ this equation can be rewritten as:

$$
\begin{array}{r}
\left.\frac{\partial \bar{\theta}}{\partial X}(0, s)\right|_{X=0}=-2 \bar{\varphi}(0, s)-s \bar{\varphi}(0, s), \text { Where } \varphi(X, 0)=0 \\
\left.\therefore \frac{\partial \bar{\theta}}{\partial X}(0, s)\right|_{X=0}=-(s+2) \bar{\varphi}(0, s)
\end{array}
$$

Comparing the two Equations (18), (20) $A$ and $B$ can be obtained as follows: 


$$
\begin{array}{r}
A \sqrt{s(s+2)}-B \sqrt{s(s+2)}=-(s+2) \bar{\varphi}(0, s) \\
(A-B)=-\frac{\sqrt{s(s+2)}}{s} \bar{\varphi}(0, s) \text { or: }(A-B)=-\sqrt{\frac{s+2}{s}} \bar{\varphi}(0, s)
\end{array}
$$

Multiplying both sides by $\mathrm{e}^{\sqrt{s(s+2)} X_{d}}$ then by $\mathrm{e}^{-\sqrt{s(s+2)} X_{d}}$ respectively one gets:

$$
\begin{gathered}
A \mathrm{e}^{\sqrt{(s+2)} X_{d}}-B \mathrm{e}^{\sqrt{s(s+2)} X_{d}}=-\bar{\varphi}(0, s) \sqrt{\frac{s+2}{s}} \mathrm{e}^{\sqrt{s(s+2)} X_{d}} \\
A \mathrm{e}^{-\sqrt{s(s+2)} X_{d}}-B \mathrm{e}^{-\sqrt{s(s+2)} X_{d}}=-\bar{\varphi}(0, s) \sqrt{\frac{s+2}{s}} \mathrm{e}^{-\sqrt{s(s+2)} X_{d}}
\end{gathered}
$$

Subtracting (19), (21) one gets:

$$
B\left[\mathrm{e}^{+\sqrt{s(s+2)} X_{d}}-\mathrm{e}^{-\sqrt{s(s+2)} X_{d}}\right]=\bar{\varphi}(0, s) \sqrt{\frac{s+2}{s}} \mathrm{e}^{+\sqrt{s(s+2)} X_{d}}
$$

Thus:

$$
B=\frac{\bar{\varphi}(0, s) \sqrt{\frac{s+2}{s}} \mathrm{e}^{+\sqrt{s(s+2)} X_{d}}}{2 \sinh \sqrt{s(s+2)} X_{d}}
$$

Also from (19), (22) one gets:

$$
A\left[\mathrm{e}^{+\sqrt{s(s+2)} X_{d}}-\mathrm{e}^{-\sqrt{s(s+2)} X_{d}}\right]=\bar{\varphi}(0, s) \sqrt{\frac{s+2}{s}} \mathrm{e}^{+\sqrt{s(s+2)} X_{d}}
$$

Thus:

$$
A=\frac{\bar{\varphi}(0, s) \sqrt{\frac{s+2}{s}} \mathrm{e}^{-\sqrt{s(s+2)} X_{d}}}{2 \sinh \sqrt{s(s+2)} X_{d}}
$$

Substituting in Equation (17) one gets:

$$
\bar{\vartheta}(X, s)=\frac{\bar{\varphi}(0, s) \sqrt{\frac{s+2}{s}}\left[\mathrm{e}^{+\sqrt{s(s+2)}\left(X_{d}-X\right)}+\mathrm{e}^{-\sqrt{s(s+2)}\left(X_{d}-X\right)}\right]}{\mathrm{e}^{\sqrt{s(s+2)} X_{d}}\left[1-\mathrm{e}^{-2 \sqrt{s(s+2)} X_{d}}\right]}
$$

Considering that: $\frac{1}{1-a}=\sum_{n=0}^{\infty} a^{n},|a|<1 \quad$ [39]

Thus:

$$
\frac{1}{\left[1-\mathrm{e}^{-2 \sqrt{s(s+2)} X_{d}}\right]}=\sum_{n=0} \mathrm{e}^{-2 n \sqrt{s(s+2)} X_{d}}
$$

This gives:

$$
\bar{\vartheta}(X, s)=\bar{\varphi}(0, s) \sqrt{\frac{s+2}{s}}\left[\sum_{n=0}^{\infty} \mathrm{e}^{-\left(X+2 n X_{d}\right) \sqrt{s(s+2)}}+\sum_{n=0}^{\infty} \mathrm{e}^{-\left(2(1+n) X_{d}-X\right) \sqrt{s(s+2)}}\right]
$$

Neglecting the last term with respect to the first one, one gets:

$$
\bar{\vartheta}(X, s)=\sum_{n=0}^{\infty} \bar{\varphi}(0, s) \frac{s+2}{\sqrt{s(s+2)}} \mathrm{e}^{-\left(X+2 n X_{d}\right) \sqrt{s(s+2)}}
$$


This equation can be rewritten in the form:

$$
\begin{aligned}
\bar{\vartheta}(X, s)= & \sum_{n=0}^{\infty}\left[\bar{\varphi}(0, s) \frac{s}{\sqrt{s(s+2)}} \mathrm{e}^{-\left(X+2 n X_{d}\right) \sqrt{s(s+2)}}\right. \\
& \left.+\bar{\varphi}(0, s) \frac{2}{\sqrt{s(s+2)}} \mathrm{e}^{-\left(X+2 n X_{d}\right) \sqrt{s(s+2)}}\right]
\end{aligned}
$$

Let, $f(s)=\frac{1}{\sqrt{s(s+2)}} \mathrm{e}^{-\left(X+2 n X_{d}\right) \sqrt{s(s+2)}}$ we get:

$$
\bar{\vartheta}(X, s)=\sum_{n=0}^{\infty}[\bar{\varphi}(0, s)\{s f(s)\}+2 \bar{\varphi}(0, s) f(s)]
$$

Put :

$$
2 \bar{\varphi}(0, s) f(s)=M_{1}, \quad \bar{\varphi}(0, s)\{s f(s)\}=M_{2}
$$

From Equation (9):

$$
\begin{gathered}
L^{-1}\{\bar{\varphi}(s)\}=\frac{q(t)(1-R)}{W \rho c_{p}\left(T_{m}-T_{0}\right)} \\
L^{-1}\{f(s)\}=L^{-1}\left\{\frac{1}{\sqrt{s(s+2)}} \mathrm{e}^{-\left(X+2 n X_{d}\right) \sqrt{s(s+2)}}\right\}
\end{gathered}
$$

We have: $[40]$

$$
L^{-1} \frac{\left.\mathrm{e}^{-c\left[(s+a) \frac{1}{2}(s+b) \frac{1}{2}\right.}\right]}{(s+a)^{\frac{1}{2}}(s+b)^{\frac{1}{2}}}=\mathrm{e}^{-\left(\frac{a+b}{2}\right) \tau} I_{0}\left[\left(\frac{a-b}{2}\right)\left(\tau^{2}-c^{2}\right)^{\frac{1}{2}}\right], \tau>c, c>0
$$

Put: $c=X+2 n X_{d}, b=0, a=2, \tau=\frac{t}{2 t_{k}}$ one gets:

$$
L^{-1} \frac{\mathrm{e}^{-\left(X+2 n X_{d}\right) \sqrt{s(s+2)}}}{\sqrt{s(s+2)}}=\mathrm{e}^{-\tau} I_{0} \sqrt{\tau^{2}-c^{2}}
$$

The modified Bessel function $I_{\eta}(x)$ is written in the following form: [41]

$$
I_{\eta}(x)=\sum_{m=1}^{\infty} \frac{\left(\frac{x}{2}\right)^{(\eta+2 m)}}{m !} \Gamma(\eta+m+1)
$$

Comparing (25), (26) (at $\eta=0$ ) one gets:

$$
L^{-1}\left\{f_{s}\right\}=\mathrm{e}^{-\tau} \sum_{m=1}^{\infty} \frac{\left[\tau^{2}-\left(X+2 n X_{d}\right)^{2}\right]^{m}}{2^{2 m} m !} \Gamma(m+1)
$$

Thus the inverse in time domain is as follows:

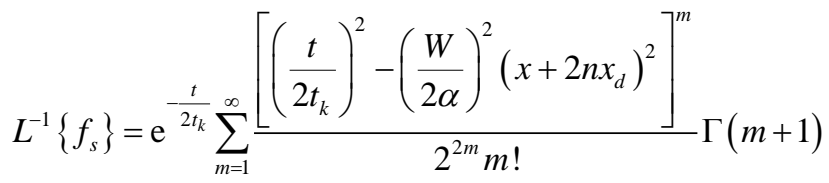


where: $\Gamma(m+1)=m$ !

$$
L^{-1}\{\bar{\varphi}(s)\}=\frac{q(t)}{W \rho c_{p}\left(T_{m}-T_{0}\right)}
$$

The convolution theorem:

$$
L^{-1}\left[f_{1}(s) f_{2}(s)\right]=\int_{0}^{t} F_{1}(t-u) F_{s}(u) \mathrm{d} u
$$

Thus

$$
\begin{aligned}
L^{-1}\left[M_{1}\right] & =L^{-1}[2 \varphi(s) f(s)] \\
& =2 \int_{0}^{t} \frac{q(t-u)}{W \rho c_{p}\left(T_{m}-T_{0}\right)} \mathrm{e}^{-\frac{u}{2 t_{k}} \sum_{m=1}^{\infty}} \frac{\left[\left(\frac{u}{2 t_{k}}\right)^{2}-\left(\frac{W}{2 \alpha}\right)^{2}\left(x+2 n x_{d}\right)^{2}\right]^{m}}{2^{2 m}}
\end{aligned}
$$

Also we have:

$$
L\left[\frac{\mathrm{d}}{\mathrm{d} t} F(t)\right]=s f(s)-f(0),
$$

For such a case one has $f(0)=0$, thus:

$$
\begin{aligned}
& L^{-1}\left[M_{2}\right]=L^{-1}[\bar{\varphi} s f(s)]=\int_{0}^{t} \varphi(t-u) \frac{\mathrm{d}}{\mathrm{d} u} f(u) \mathrm{d} u
\end{aligned}
$$

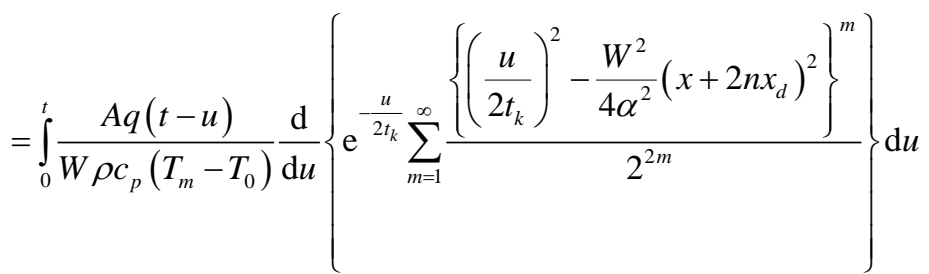

$$
\begin{aligned}
& L^{-1}[\bar{\vartheta}(X, s)]=L^{-1}\left[M_{1}\right]+L^{-1}\left[M_{2}\right]
\end{aligned}
$$

$\therefore \vartheta(x, t)$

$$
\begin{aligned}
& =\sum_{n=1}^{\infty}\left[\left(2-\frac{1}{2 t_{k}}\right)^{t} \int_{0}^{t} \frac{A q(t-u)}{W \rho c_{p}\left(T_{m}-T_{0}\right)} \mathrm{e}^{-\frac{u}{2 t_{k}} \sum_{m=1}^{\infty}} \frac{\left\{\left(\frac{u}{2 t_{k}}\right)^{2}-\frac{W^{2}}{4 \alpha^{2}}\left(x+2 n x_{d}\right)^{2}\right\}^{m}}{2^{2 m}} \mathrm{~d} u\right. \\
& \left.+\int_{0}^{t} \frac{A q(t-u)}{W \rho c_{p}\left(T_{m}-T_{0}\right)} \frac{1}{2 t_{k}^{2}} \mathrm{e}^{-\frac{u}{2 t_{k}}} \sum_{m=1}^{\infty} \frac{m\left\{\left(\frac{u}{2 t_{k}}\right)^{2}-\frac{W^{2}}{4 \alpha^{2}}\left(x+2 n x_{d}\right)^{2}\right\}^{m-1}}{2^{2 m}} u \mathrm{~d} u\right]
\end{aligned}
$$

where: $q(t-u)=q_{\max } \mathrm{e}^{-\left(\frac{(t-u)-t_{0}}{\gamma}\right)^{2}}, q_{\max }, \mathrm{W} / \mathrm{m}^{2}$ is the laser maximum power density

At $x=0$ we get: 


$$
\begin{aligned}
& \vartheta(0, t) \\
& =\sum_{n=1}^{\infty}\left[\left(2-\frac{1}{2 t_{k}}\right)_{0}^{t} \frac{A q(t-u)}{W \rho c_{p}\left(T_{m}-T_{0}\right)} \mathrm{e}^{-\frac{u}{2 t_{k}} \sum_{m=1}^{\infty} \frac{\left\{\left(\frac{u}{2 t_{k}}\right)^{2}-\frac{W^{2}}{4 \alpha^{2}}\left(2 n x_{d}\right)^{2}\right\}^{m}}{2^{2 m}} \mathrm{~d} u}\right. \\
& \left.+\int_{0}^{t} \frac{A q(t-u)}{W \rho c_{p}\left(T_{m}-T_{0}\right)} \frac{1}{2 t_{k}^{2}} \mathrm{e}^{-\frac{u}{2 t_{k}} \sum_{m=1}^{\infty}} \frac{m\left\{\left(\frac{u}{2 t_{k}}\right)^{2}-\frac{W^{2}}{4 \alpha^{2}}\left(2 n x_{d}\right)^{2}\right\}^{m-1}}{2^{2 m}} u \mathrm{~d} u\right]
\end{aligned}
$$

\section{Computations}

The obtained thermal profile is computed for different laser pulses with different maximum power densities as follows:

$$
q_{\max }=2 \mathrm{E} 7,1.5 \mathrm{E} 7,1 \mathrm{E} 7,0.8 \mathrm{E} 7,0.7 \mathrm{E} 7,0.6 \mathrm{E} 7,0.5 \mathrm{E} 7,0.4 \mathrm{E} 7,0.3 \mathrm{E} 7,0.2 \mathrm{E} 7, \mathrm{~W} / \mathrm{m}^{2} .
$$

The laser pulse shape is taken of Gaussian form as: $q(t)=q_{\max } \mathrm{e}^{-\left(\frac{t-t_{0}}{\gamma}\right)^{2}}, \gamma$ is the full width at half maximum of the suggested pulse, $t_{0}$ in seconds is the time required for $q(t)$ to reach the maximum value $q_{\max }$.

The other laser pulse parameters are as follows: $t_{0}=6 \mu \mathrm{sec}, \gamma=6 \mu \mathrm{sec}$. pulse duration $=12 \mu \mathrm{sec}$, the slab thickness $=300 \mu \mathrm{m}$, the absorption coefficient $A=$ $0.67, t_{k}=1 \mu \mathrm{sec}$.

The physical and thermal properties of the silver selenide slab material [42] [43] are given in Table 1.

The temperature of the irradiated front surface $\vartheta(0, t)$ for the different pulses is computed and are illustrated graphically in Figure 1 and Figure 2 and are tabulated in Table 2 for $m=1, n=1$, and in Table 3 for $m=1, n=1.2$.

The critical time $t_{p h}$ required to start phase transition, and the critical time $t_{m}$ required to initiate melting at the front surface are obtained for the considered pulses and are tabulated in Table 4 for $m=1, n=1$, and in Table 5 for $m=1$, $n=1.2$ and are illustrated graphically in Figure 3 and Figure 4 .

\section{Conclusions}

As a result of the study the following conclusions can be made:

1) The temperature of the irradiated surface does depend linearly on the maximum power density $q_{\max }$ of the laser pulse and also on the absorption coefficient $(1-R)$.

2) The dependence on the slab thickness and the pulse parameters $\gamma \& t_{0}$ are no longer linear.

3) The dependence of the $t_{p h}$ and $t_{m}$ on $q_{\max }$ is not linear. 
Table 1. The physical and thermal properties of the silver selenide slab material [42] [43].

\begin{tabular}{ccccccc}
\hline \multirow{2}{*}{ Silver Selenide } & $\rho, \mathrm{kg} / \mathrm{m}^{3}$ & $\lambda, \mathrm{W} / \mathrm{mK}$ & $\alpha, \mathrm{m}^{2} / \mathrm{sec}$ & $c_{p}, \mathrm{j} / \mathrm{kgK}$ & $T_{m}, \mathrm{~K}$ & $T_{p h}, \mathrm{~K}$ \\
\cline { 2 - 7 } & 8200 & 1.08 & $3.9 \mathrm{E}-7$ & 277 & 855 & 403 \\
\hline
\end{tabular}

Table 2. The temperature of the front surface as a function of the $q_{\max }, \mathrm{W} / \mathrm{m}^{2}$ and exposure time, $(m=1, n=1)$.

\begin{tabular}{|c|c|c|c|c|c|c|c|c|c|c|}
\hline \multirow{2}{*}{$t, \mathrm{~ms}$} & \multirow[b]{2}{*}{$2 \times 10^{7}$} & \multirow[b]{2}{*}{$1.5 \times 10^{7}$} & \multirow[b]{2}{*}{$1 \times 10^{7}$} & \multirow[b]{2}{*}{$0.8 \times 10^{7}$} & \multirow[b]{2}{*}{$0.7 \times 10^{7}$} & \multirow[b]{2}{*}{$0.6 \times 10^{7}$} & \multirow[b]{2}{*}{$0.5 \times 10^{7}$} & \multirow[b]{2}{*}{$0.4 \times 10^{7}$} & \multirow[b]{2}{*}{$0.3 \times 10^{7}$} & \multirow[b]{2}{*}{$0.2 \times 10^{7}$} \\
\hline & & & & & & & & & & \\
\hline 2 & 325.1 & 243.83 & 162.55 & 130.04 & 113.78 & 97.53 & 81.27 & 65.02 & 48.76 & 32.51 \\
\hline 3 & 474.14 & 355.61 & 237.07 & 189.66 & 165.95 & 142.24 & 118.54 & 94.83 & 71.12 & 47.41 \\
\hline 4 & 613.78 & 460.34 & 306.89 & 245.51 & 214.82 & 184.134 & 153.44 & 122.75 & 92.06 & 61.38 \\
\hline 6 & 829.56 & 622.17 & 414.78 & 331.82 & 290.35 & 248.86 & 207.39 & 165.91 & 124.43 & 82.96 \\
\hline 7 & 885.56 & 664.17 & 442.78 & 354.22 & 309.95 & 265.67 & 221.39 & 177.11 & 132.83 & 88.56 \\
\hline 8 & 998.12 & 748.59 & 499.06 & 399.25 & 314.34 & 499.06 & 224.53 & 179.62 & 134.72 & 89.81 \\
\hline 9 & 867.2 & 650.4 & 433.6 & 346.88 & 303.52 & 260.16 & 216.8 & 173.44 & 130.08 & 86.72 \\
\hline 10 & 798.52 & 598.89 & 399.26 & 319.41 & 279.73 & 239.56 & 199.81 & 159.84 & 119.88 & 79.92 \\
\hline 12 & 590.88 & 443.16 & 295.44 & 236.35 & 206.81 & 177.26 & 147.72 & 118.18 & 88.63 & 59.09 \\
\hline
\end{tabular}

Table 3. The temperature of the front surface as a function of the $q_{\max }, \mathrm{W} / \mathrm{m}^{2}$ and exposure time, $(m=1, n=1,2)$

\begin{tabular}{|c|c|c|c|c|c|c|c|c|c|c|}
\hline$q_{\max }$ & $2 \times 10^{7}$ & $1.5 \times 10^{7}$ & $1 \times 10^{7}$ & $0.8 \times 10^{7}$ & $0.7 \times 10^{7}$ & $0.6 \times 10^{7}$ & $0.5 \times 10^{7}$ & $0.4 \times 10^{7}$ & $0.3 \times 10^{7}$ & $0.2 \times 10^{7}$ \\
\hline 2 & 1625.48 & 1219.11 & 812.74 & 650.19 & 568.92 & 487.64 & 406.37 & 325.10 & 243.82 & 162.55 \\
\hline 3 & 2370.70 & 1778.03 & 1185.35 & 948.28 & 829.75 & 711.21 & 592.68 & 474.14 & 355.61 & 237.07 \\
\hline 4 & 3068.86 & 2301.65 & 1534.43 & 1227.54 & 1074.10 & 920.66 & 767.22 & 613.77 & 460.33 & 306.89 \\
\hline 6 & 4147.78 & 3110.84 & 2073.89 & 1659.11 & 1451.72 & 1244.33 & 1036.95 & 829.56 & 622.17 & 414.78 \\
\hline 7 & 4427.18 & 3320.84 & 2213.89 & 1771.11 & 1549.72 & 1328.33 & 1106.94 & 885.56 & 664.17 & 442.78 \\
\hline 8 & 4590.58 & 3442.94 & 2295.29 & 1836.23 & 1606.70 & 1377.17 & 1147.65 & 918.12 & 688.59 & 459.06 \\
\hline 9 & 4335.48 & 3251.99 & 2167.99 & 1734.39 & 1517.59 & 1300.79 & 1083.99 & 867.20 & 680.40 & 433.60 \\
\hline 10 & 3992.6 & 2994.45 & 1996.30 & 1597.04 & 1397.41 & 1197.78 & 998.15 & 798.52 & 598.89 & 399.26 \\
\hline 12 & 2954.36 & 2215.77 & 1477.18 & 1181.74 & 1034.03 & 886.31 & 738.59 & 590.87 & 443.15 & 295.44 \\
\hline
\end{tabular}

Table 4. The critical time $t_{p h}$ required to start phase transition, and the critical time $t_{m}$ required to initiate melting at the front surface are obtained for the considered pulses for $m=1, n=1$.

\begin{tabular}{ccc}
\hline$q_{\max }, \mathrm{W} / \mathrm{m}^{2}$ & $t_{p h}, \mu \mathrm{sec}$ & $t_{m}, \mu \mathrm{sec}$ \\
\hline $2 \times 10^{7}$ & 2.7 & 6.3 \\
$1.5 \times 10^{7}$ & 3.7 & - \\
$1 \times 10^{7}$ & 5.7 & - \\
$0.8 \times 10^{7}$ & 8 & - \\
\hline
\end{tabular}


Table 5. The critical time $t_{p h}$ required to start phase transition, and the critical time $t_{m}$ required to initiate melting at the front surface are obtained for the considered pulses for $m=1, n=1,2$.

\begin{tabular}{ccc}
\hline$q_{\max }, \mathrm{W} / \mathrm{m}^{2}$ & $t_{p h}, \mu \mathrm{sec}$ & $t_{m,}, \mu \mathrm{sec}$ \\
\hline $2 \times 10^{7}$ & 0.6 & 1.3 \\
$1.5 \times 10^{7}$ & 0.8 & 1.5 \\
$1 \times 10^{7}$ & 1 & 2.3 \\
$0.8 \times 10^{7}$ & 1.3 & 2.7 \\
$0.6 \times 10^{7}$ & 1.7 & 3.7 \\
$0.4 \times 10^{7}$ & 2.6 & 6.5 \\
$0.2 \times 10^{7}$ & 5.5 & - \\
\hline
\end{tabular}

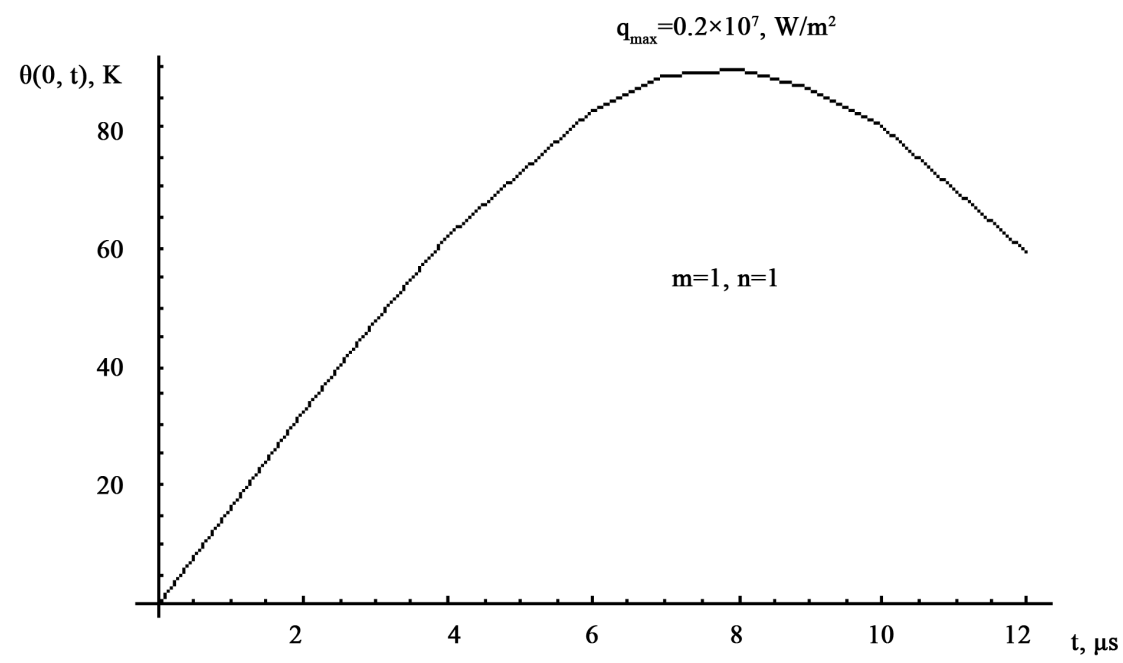

Figure 1. The temperature of the irradiated front surface as a function of the laser exposure time " $t$ ". $q_{\max }=0.2 \times 10^{7} \mathrm{~W} / \mathrm{m}^{2}, m=1$ and $n=1$.

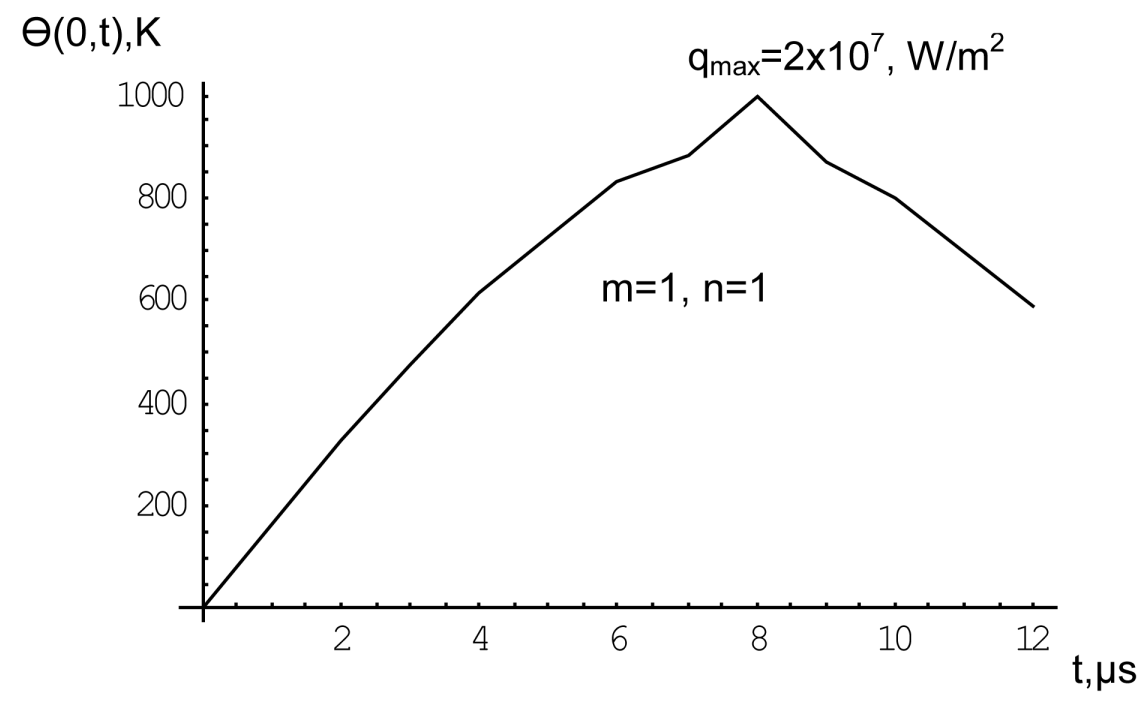

Figure 2. The temperature of the irradiated front surface as a function of the laser exposure time " $t$ ". $q_{\max }=0.2 \times 10^{7} \mathrm{~W} / \mathrm{m}^{2}, m=1$ and $n=1$. 


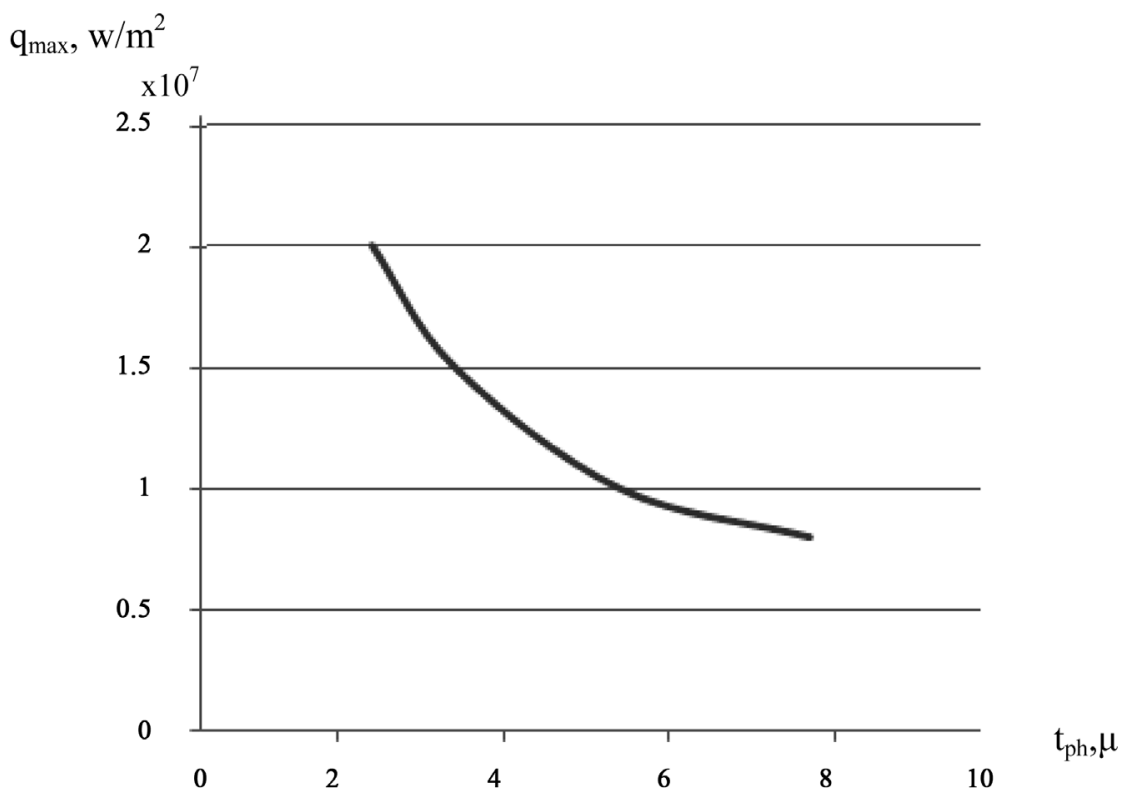

Figure 3. The critical time $t_{p h}$ required to start phase transition as a function of the maximum power density of the laser pulse for $m=1$ and $n=1$. The other parameters are kept constant.

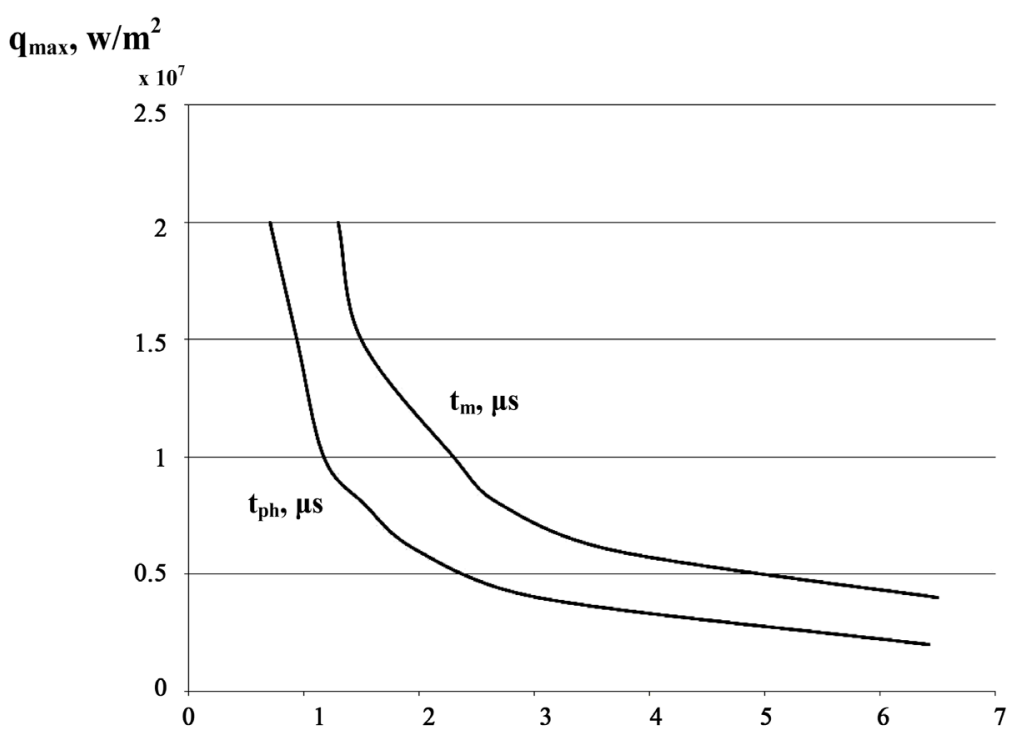

Figure 4. The critical time $t_{p h}$ required to start phase transition and the critical time $t_{m}$ required to initiate melting as a function of the maximum power density of the laser pulse for $m=1$ and $n=1,2$. The other parameters are kept constant.

4) Successive pulses may be required to initiate phase transition or melting.

5) For the summation over " $m$ " and " $n$ " one gets acceptable values for $\theta(0, t)$ only for $m=1$.

But for $m>1$ one gets negative values.

Increasing values of $\theta(0, t)$ are obtained with increasing $n$ values. 
6) The extension of the present technique to other materials makes it possible to specify the optimum operation conditions to attain and maintain a certain phase for specific medical and technological applications.

\section{References}

[1] Ready, J.F. (1965) Effects Due to Absorption of Laser Radiation. Journal of Applied Physics, 36, 462-468. https://doi.org/10.1063/1.1714012

[2] Ready, J.F. (1978) Industrial Applications of Laser. Academic Press, New York.

[3] He, X. and Debroy, T. (2003) Probing Temperature during Laser Spot Welding from Vapor Composition and Modeling. Journal of Applied Physics, 94, 6949-6958. https://doi.org/10.1063/1.1622118

[4] Zhang, Y.M., Shen, Z.H. and Ni, X.W. (2014) Modeling and Simulation on Long Pulse Laser Drilling Processing. International Journal of Heat and Mass Transfer, 73, 429-437. https://doi.org/10.1016/j.ijheatmasstransfer.2014.02.037

[5] Zhou, B.H. and Mahdavian, S.M. (2004) Experimental and Theoretical Analyses of Cutting Nonmetallic Materials by Low Power $\mathrm{CO}_{2}$-Laser. Journal of Materials Processing, 146, 188-192. https://doi.org/10.1016/j.jmatprotec.2003.10.017

[6] Liu, B.A., Hu, G.H., Zhao, Y.A., Zhang, L.S., Sun, X., Wang, Z.P. and Xu, X.G. (2013) Laser Induced Damage of DKDP Crystals with Different Deuterated Degrees. Optics \& Laser Technology, 45, 469-472. https://doi.org/10.1016/j.optlastec.2012.06.008

[7] Abd El-Gany, S.E.-S. (2009) A Theoretical Study on the Melting of a Finite Slab with a Pulsed Laser. Optik, 120, 890-897. https://doi.org/10.1016/j.ijleo.2008.04.002

[8] Detlev, R., Marco, J. and Kai S., (2009) Laser Damage Thresholds of Optical Coatings. Thin Solid Films, 518, 1607-1613. https://doi.org/10.1016/j.tsf.2009.07.150

[9] Bo F., Dayon Z.,Fei L.,Chenxing L.,Guodong L., (2012) Pulsed Laser Induced Damage in SOI Material. Journal of Physics and Chemistry of Solids, 73, 969-972. https://doi.org/10.1016/j.jpcs.2012.03.002

[10] Hassan, A.F., El-Niclawy, M.M., El-Adawi, M.K. and Hemida, A.A. (1993) A General Problem of Pulse Heating Of a Slab. Optics \&Laser Technology, 25, 155-162. https://doi.org/10.1016/0030-3992(93)90059-O

[11] El-Adawi, M.K., Abdel-Naby, M.A. and Shalaby, S.A. (1994) Laser Heating of a Slab Having Temperature Dependent Surface Absorbtance. Vacuum, 45, 65-70. https://doi.org/10.1016/0042-207X(94)90344-1

[12] El-Adawi, M.K., Abdel-Naby, M.A. and Shalaby, S.A. (1995) Laser Heating of a Two-Layer System with Constant Surface Absorptance: An Exact Solution. International Journal Heat Mass Transfer, 38, 947-952. https://doi.org/10.1016/0017-9310(94)00162-O

[13] El-Adawi, M.K., Shalaby, S.A. and Abdel-Naby, M.A. (1995) Laser Heating of a Two-Layer System with Temperature Dependent Front Surface Absorptance. Vacuum, 64, 37-42. https://doi.org/10.1016/0042-207X(93)E0005-6

[14] Hassan A.F., El-Niclawy, M.M., El-Adawi, M.K., Nasr, A.E., Hemida M.A. and Abd El-Ghaffar, O.A. (1996) Heating Effects Induced by a Pulsed Laser in a Semi-Infinite Target in View of The theory of Linear Systems. Journal of Optics \& Laser Technology, 28, 337-343. https://doi.org/10.1016/0030-3992(95)00083-6

[15] El-Adawi, M.K., Shalaby, S.A. and Abd El-Naby, M.A. (1996) Laser Heating of a Slab with Temperature Dependent Absorptance In Relation to the Cooling Conditions. Vacuum, 47, 1167-1173. https://doi.org/10.1016/0042-207X(96)00152-2 
[16] Hassan, A.F., El-Niclawy, M.M., El-Adawi, M.K., Nasr, A.E., Hemida, M.A. and Abd El-Ghaffar, O.A. (1997) Heating a Slab of Finite Thickness with a Pulsed Laser in View of the Theory of Linear Systems. Pure and Applied Optics, 6, 67-84. https://doi.org/10.1088/0963-9659/6/1/009

[17] El-Adawi, M.K., Shalaby, S.A., Mostafa, S.S. and Kotkata, M.K. (2007) Laser Thermal Response of a Finite Slab as a Function of the Laser Pulse Parameters. Optics and Laser Technology, 39, 424-429. https://doi.org/10.1016/j.optlastec.2005.06.038

[18] El-Adawi, M.K. and Al-Theeb, H.M. (2008) Heating One-Dimensional Homogeneous Slab with Pulsed Laser in Relation to the Laser Pulse Parameters. AJSE, 33, 147-162.

[19] El-Adawi, M.K. and Al-Fanakh, H.S. (2013) Laser Heating of a Finite Silver Selenide Slab. Journal of Optics \& Laser Technology, 49, 231-236. https://doi.org/10.1016/j.optlastec.2012.12.031

[20] El-Adawi, M.K. and Al-Nuaim, I.A. (2013) Heating of a Finite Slab with CW Laser in Relation to Cooling Conditions at the Rear Surface. Material Science Research India, 10, 17-22.

[21] El-Adawi, M.K., Shalaby, S.A. and Abd El-Ghany, S.E.S. (2015) Interaction of Laser Radiation with Solids-Review Articles. International Journal of Natural Sciences Research, 3, 83-100. https://doi.org/10.18488/journal.63/2015.3.6/63.6.83.100

[22] El-Adawi, M.K. and Al-Theeb, H.M. (2015) Heating Two-Layer System with Pulsed Laser. International Journal of Science and Research, 4, 247-254.

[23] Glass, D.E., Ozisik, M.N. and Vick, B. (1985) Hyperbolic Heat Conduction with Surface Radiation. International Journal of Heat and Mass Transfer, 28, 1823-1830. https://doi.org/10.1016/0017-9310(85)90204-2

[24] Frankel, J.I., Vic, B. and Ozisk, M.N. (1987) General Formation and Analysis of Hyperbolic Heat Conduction in Composite Media. International Journal of Heat and Mass Transfer, 30, 1293-1305. https://doi.org/10.1016/0017-9310(87)90162-1

[25] Gembarovic, J. and Majernik, V. (1988) Non-Fourier Propagation of Heat Pulses in Finite Medium. International Journal of Heat and Mass Transfer, 31, 1073-1080. https://doi.org/10.1016/0017-9310(88)90095-6

[26] Kaminski, W. (1990) Hyperbolic Heat Conduction Equation for Materials with a Nonhomogeneous Inner Structure. Journal of Heat Transfer, 112, 555-560. https://doi.org/10.1115/1.2910422

[27] Lewandowska, M. (2001) Hyperbolic Heat Conduction in the Semi-Infinite Body with a Time-Dependent Laser Source. Heat and Mass Transfer, 37, 333-342. https://doi.org/10.1007/s002310000176

[28] Peshkov, V. (1944) Second Sound in Helium II. Journal of Physics, 8, 381-389.

[29] Bertman, B. and Sandiford, D.J. (1970) Second Sound in Solid Helium. Scientific American, 222, 92-101. https://doi.org/10.1038/scientificamerican0570-92

[30] Wiggert, D.C. (1970) Analysis of Early-Time Transient Heat Conduction by Method of Characteristics. Journal of Heat Transfer, 99, 35-40. https://doi.org/10.1115/1.3450651

[31] Carey, G.F. and Tsai, F.M. (1982) Hyperbolic Heat Transfer with Reflection. Numerical Heat Transfer, 5, 309-327. https://doi.org/10.1080/10407788208913451

[32] Ozisik, M.N. and Vick, B. (1984) Propagation and Reflection of Thermal Waves in a Finite Medium. International Journal of Heat and Mass Transfer, 27, 1845-1854. https://doi.org/10.1016/0017-9310(84)90166-2 
[33] Miki, H. (1991) Superlattice Formation and Phase Transition in Silver Selenide. Journal of Applied Physics, 30, 1765-1869. https://doi.org/10.1143/JJAP.30.1765

[34] Kumar, M. and Pradeep, B. (2002) Structural Electrical and Optical Properties of Silver Selenide Thin Films. Semiconductor Science and Technology, 7, 261-265. https://doi.org/10.1088/0268-1242/17/3/314

[35] Schoen, D., Yie, C. and Cui, Y. (2007) Electrical Switching and Phase Transformation in Silver Selenide Nanowiers. Journal of the American Chemical Society, 129, 4116-4117.

[36] Banus, M.D. (1965) Pressure Dependence of Alpha-Beta Transition Temperature in Silver Selenide. Science, 147, 732-733. https://doi.org/10.1126/science.147.3659.732

[37] El-Adawi, M.K. and El-Shehawy, E.F. (1986) Heating a Slab Induced by a TimeDependent Laser Irradiance-An Exact Solution. Journal of Applied Physics, 60, 2250-2255. https://doi.org/10.1063/1.337184

[38] Aivazov, A.A., Okhotin, A.S. and Gutsev, A.F. (1971) Thermal Conductivity of Silver Selenide in the Solid and Liquid States. Fizika, 4, 128-130. https://doi.org/10.1007/BF00817988

[39] Rainville, E.D. and Bedient, P.E. (1974) Elementary Differential Equations. 5th Edition, Macmillan Publishing Co., New York.

[40] Roberts, G.E. and Kauvman, H. (1966) Tables of Laplace Transforms. Saunders Company, London, 254

[41] Gradshtegn, I.S. and Ryzhik, I.M. (1980) Tables of Integrals, Series and Products. 4th Edition, Academic Press, New York.

[42] West, A.R. (1999) Basic Solid State Chemistry. John Wiley \& Sons, Hoboken.

[43] Dean, J.A. (1978) Lange's Handbook of Chemistry. McGraw Hill Book Company, New York, 126-127. 